\title{
PERIPHERAL NEUROPATHY IN BEHCET'S DISEASE: CLINICAL AND NEUROPHYSIOLOGICAL STUDY
}

\author{
By
Hossam Mohamed Emam, Mohie El Din Tharwat Mohamed, Hatem Galal Abdullah* and ELSayed Maher Arafat \\ Departments of Neurology and Rheumatology*, Faculty of Medicine, Al-Azhar \\ University, Cairo, Egypt
}

Corresponding author: ELSayed Maher Arafat, E-mail: sayed.maher008@gmail.com

\begin{abstract}
Background: Behçet's disease (BD) is a chronic idiopathic relapsing condition, whereas multiple organs are affected as a sequential effect of the vascular and inflammatory process. Based on the neurophysiological studies, $20-40 \%$ of BD patients may have silent neurological involvement (SNI) with no or very mild clinical manifestations.

Objectives: Assessing clinically and electro physiologically the prevalence of symptomatic and asymptomatic peripheral neuropathy (PN) among BD patients along with the potential predictors of PN among BD patients.

Patients and Methods: All patients with clinically established BD according to the International Criteria for Behçet's Disease (ICBD) were involved in the current study. All patients were submitted to clinical evaluation, neuropathy symptom score (NSS) along with electrophysiological nerve conduction studies including sensory and motor nerve conduction assessment.

Results: An overall 30 participants who fulfilled the inclusion criteria were enrolled in the study. There were $22(73.3 \%)$ males and $8(26.7 \%)$ females with a mean age of $37.9 \pm 8.31$ years. Based on the electrophysiological studies, $16(53.3 \%)$ patients had the evidence of peripheral neuropathy in the terms of mixed (23.3\%), motor (6.6\%), and sensory (23.3\%) nerve affection. The most affected peripheral nerve was sural nerve (46.6\%) succeeded by peroneal (43.3\%) and ulnar (20\%) nerves. The univariate regression model showed that the age of the patients and the duration of the disease were independent predictors of peripheral neuropathy.
\end{abstract}

Conclusion: $\mathrm{PN}$ is a considerable complication of $\mathrm{BD}$, whereby health care providers should employ a routine follow up protocol for early detection of even the subclinical PN.

Keywords: Behçet's disease, peripheral neuropathy, electrophysiological studies.

\section{INTRODUCTION}

Behçet's disease (BD) is a chronic idiopathic relapsing condition, whereas multiple organs are affected as a sequential effect of the vascular and inflammatory process (Emmi et al., 2018). The burden of BD differed geographically, whereby countries along the silk road, apart from Japan to the Mediterranean area, showed a high prevalence of BD (Uygunoglu et al., 2018). Subsequently, BD patients usually presented in the third decade of life with an equal proportion of males and females; however, males often showed more 
progressive disease course (Mazzoccoli et al., 2016).

Of note, BD is associated with abundant clinical manifestations counterpart eye inflammation, mucocutaneous lesions, vasculopathy, cardiac, pulmonary, musculoskeletal, gastrointestinal and neurological insults (Yusuf Yazici et al., 2009). The diagnosis of such condition is based mainly on the clinical evaluation according to the International Criteria for Behçet's Disease (ICBD), whereas the serological assessment is usually limited in the diagnosis of BD (Siva and Saip, 2009).

Approximately, 5-10 years after the onset of BD, Neuro-Behçet's syndrome (NBS) could be evolved among $4-50 \%$ of BD patients, whereby males had a triple risk of affection relative to females ( $\mathrm{Al}$ Araji and Kidd, 2009). Interestingly, nearly $5-20 \%$ of the patients are presenting with a neurological insult as the initial presentation instead of oral ulcers which is the predominant presentation of BD (Siva and Saip, 2009). Unfortunately, the involvement of the nervous system carries bad prognosis (Dutra et al., 2012).

In contrast, primary neurological insult includes cerebral venous sinus thrombosis, headache, arterial NBS, intraaxial NBS, subclinical NBS, (PN) (Kalra et al., 2014). Subsequent to that, secondary neurological insult occurred as an eventual effect of the systemic consequences of $\mathrm{BD}$ such as cerebral embolic events from cardiac affection and the neurological manifestations related to BD treatment (Uygunoglu et al., 2018).

The burden of PN among BD patients is deeply rare (Kalra et al., 2014).
Noteworthy, some studies revealed the existence of mononeuritis multiplex, Polyradiculoneuritis, and axonal sensory neuropathy in BD. Besides, polyneuropathy may exist as a side effect of thalidomide or colchicine (Saip et al., 2014).

Based on the neurophysiological studies, $20-40 \%$ of BD patients may have silent neurological involvement (SNI) with no or very mild clinical manifestations (Yesilot et al., 2006). Those patients may experience overt neurological manifestations within the course of the disease (Graham et al., 2013).

The current study aimed to assess clinically and electrophysiologically the prevalence of symptomatic and asymptomatic PN among BD patients along with the potential predictors of PN among BD patients.

\section{SUBJECTS AND METHODS}

This study was implemented along with the ethical committee guidelines of the Faculty of Medicine, Al-Azhar University, Cairo, Egypt. All clinical interventions were illustrated to all participants prior to study implementation. A written informed consent after a clear explanation of all study steps was obtained.

This was an observational retrospective case-control study which was carried out at the inpatient departments and outpatient clinics of Al-Azhar Neurology and Rheumatology departments at Al-Azhar University Hospitals (Al Hussein and Bab Al-sharia) from January 2019 to end of June 2019. 
Thirty patients with clinically established Behçet's disease according to the International Criteria for Behçet's Disease (ICBD) were involved in the current study. On the contrary, patients with BD and concomitant other neurological or chronic medical systemic illness that can develop or affect peripheral nerves, such as hereditary ataxias, diabetes mellitus, renal or hepatic failure were excluded from the study. Similar to that, patient subjected to substances or drugs abuse that may alter peripheral nerves like alcohol abuse, or patients with a possible mechanical nerve compression were omitted.

All patients were subordinated to meticulous history taking comprised age, sex, history of chronic diseases, drug abuse, medications, or other neurological diseases coupled with history of the present neurological manifestations to reveal symptoms of $\mathrm{BD}$ and peripheral neuropathy. Subsequent to that, general and neurological examinations were done to retrieve the clinical evidence of motor, sensory, or autonomic systems affection along with cranial nerves involvement.

Neuropathic symptoms and signs had been assessed based upon the neuropathy symptom score (NSS). The NSS is five multiple choice questions for measuring the severity of neuropathic symp-toms and signs. The NSS has a maximum score of 9, with a higher score indicating more severe neuropathic symptoms.

Electrophysiological studies of both upper and lower limbs were implemented at the room temperature, via Nihon Kochden electromyography machine, using surface electrodes for stimulation and recording.
Sensory nerve conduction was assessed based on the antidromic method. Digit2wrist and Digit5-wrist segments were used for median and ulnar nerves conduction studies, respectively. To assess the sural nerve conduction, the recording electrode was positioned posterior to the lateral malleolus, whereas the reference electrode was placed approximately $3 \mathrm{~cm}$ distally.

The recording electrodes were positioned above the abductor pollicis brevis and the adductor digiti minimi muscles for the assessment of the median and ulnar nerves, respectively. In addition, to assess the peroneal nerve, these electrodes were placed over the extensor digitorum brevis muscle, whilst they were positioned over the abductor hallucis brevis muscle to assess the tibial nerve. Generally, the active recording electrode was laid on the belly of the muscle, whereas the reference electrode was positioned at the insertion of the muscle tendon.

A neuropathy was classified as predominantly or initially involving the Schwann cell, causing myelinopathy, or involving the axon itself causing Axonopathy.

The ESTEEM (European Standardized Telematic tool to Evaluate Electrodiagnostic Methods) was used as a guideline criterion for pathophysiological interpretation of individual electrodiagnostic nerve tests and classification of polyneuropathy (Tankisi et al., 2005).

\section{Statistical Analysis:}

Continuous normally distributed data were illustrated in the form of mean, and standard deviation (SD), whereby non- 
normally distributed data were presented using median and range. Categorical variables were expressed using the number and percentage and its particular groups were compared using Pearson's chi-square test with Fisher's exact test. Furthermore, correlation analysis was conducted using Spearman's rank correlation coefficient for non-parametric variables. The univariate regression model was accomplished to elucidate the independent predictors of peripheral neuropathy using $t$-test analysis. The significance is established when $\mathrm{P}<0.05$.

Statistical analysis was executed using SPSS software version 23 for Windows (SPSS Inc., Chicago, IL, USA), and MedCalc software version 14.8 (MedCalc Software, Mariakerke, Belgium).

\section{RESULTS}

\section{Patient's demographic characteristics}

An overall 30 patients who fulfilled the inclusion criteria were recruited in the study. There were $22(73.3 \%)$ males and 8 $(26.7 \%)$ females with a mean age of $37.9 \pm 8.31$ years. There were $22(73.3 \%)$ patients had oral ulcers. Additionally, there were $20(66.7 \%), 15(50 \%), 20$ $(66.7 \%)$, and $17(56.7 \%)$ patients suffered from eye affection, skin lesions, genital ulcers, and neurological manifestations, respectively. Subsequent to that, there were $8(26.6 \%)$ patients experienced gastrointestinal manifestations, whereas 5 $(16.7 \%)$ and $3(10 \%)$ patients experienced joint affection and major vessels affection, respectively. The duration of symptoms extended from a month up to 12 months. The mean Behcet Disease Current Activity Form (BDCAF) score was $5.26 \pm 1.99$ (Table 1).

Table (1); Demographic and clinical characteristics in Behcet disease patients

\begin{tabular}{|l|l|}
\hline BDCAF score & $5.26 \pm 1.99$ \\
\hline Duration of Symptoms & $3(1-12)$ years \\
\hline Oral Ulcers & $22(73.3 \%)$ \\
\hline Genital Ulcers & $20(66.7 \%)$ \\
\hline Eye affection & $20(66.7 \%)$ \\
\hline Neurological Manifestations & $17(56.7 \%)$ \\
\hline Skin Affection & $15(50 \%)$ \\
\hline Headache & $13(43.3 \%)$ \\
\hline Gastro-intestinal Manifestations & $8(26.7 \%)$ \\
\hline Joint affection & $5(16.7 \%)$ \\
\hline Major vessels affection & $3(10 \%)$ \\
\hline BDCAF = Behcet Disease Current Activity Form \\
\hline
\end{tabular}

Clinically, $7(23.3 \%)$ of the included patients revealed symptoms of peripheral neuropathy counterpart numbness, tingling, reduced sensation, or motor weakness. On the contrary, based on the electrophysiological studies, $16(53.3 \%)$ patients had the evidence of PN in the terms of mixed 7 $(23.3 \%)$ patients, motor $2(6.6 \%)$ patients, and sensory $7(23.3 \%)$ patients nerve affection. In details, there were $5(15.6 \%), 6(20 \%)$, and 
PERIPHERAL NEUROPATHY IN BEHCET'S DISEASE: CLINICAL...

$5(16.6 \%)$ patients had median, ulnar, and tibial nerve affection, respectively. Consequently, 13 (43.3\%) patients had peroneal nerve affection, whereby 14 (46.6\%) patients had sural nerve affection (Table 2) .

Table (2): Nerve conduction study findings among Behçet's disease patients

\begin{tabular}{|l|l|l|}
\hline Sural nerve affection & $14(46.6 \%)$ & $7(23.3 \%)$ \\
\hline \multirow{2}{*}{ Peroneal nerve affection } & Axonal & $6(20 \%)$ \\
\cline { 2 - 3 } & Demyelination & $3(10 \%)$ \\
\hline \multirow{2}{*}{ Tibial nerve affection } & Axonal & $2(6.7 \%)$ \\
\cline { 2 - 3 } & Demyelination & $3(10 \%)$ \\
\hline \multirow{2}{*}{ Ulnar nerve affection } & Axonal & $2(6.7 \%)$ \\
\cline { 2 - 3 } & Demyelination & $3(10 \%)$ \\
\cline { 2 - 3 } & Axonal & $3(10 \%)$ \\
\cline { 2 - 3 } & Demyelination & \\
\hline
\end{tabular}

Out of the included patients, 9 patients were treated by colchicine. Of them, sex (66.6\%) patients experienced peripheral neuropathy apart from mixed neuropathy (4 patients), and sensory (2 patient). In contrast, sural and peroneal nerves were affected in six $(66.6 \%)$ and $5(55.5 \%)$ patients, respectively (Figure1 and Table 3).

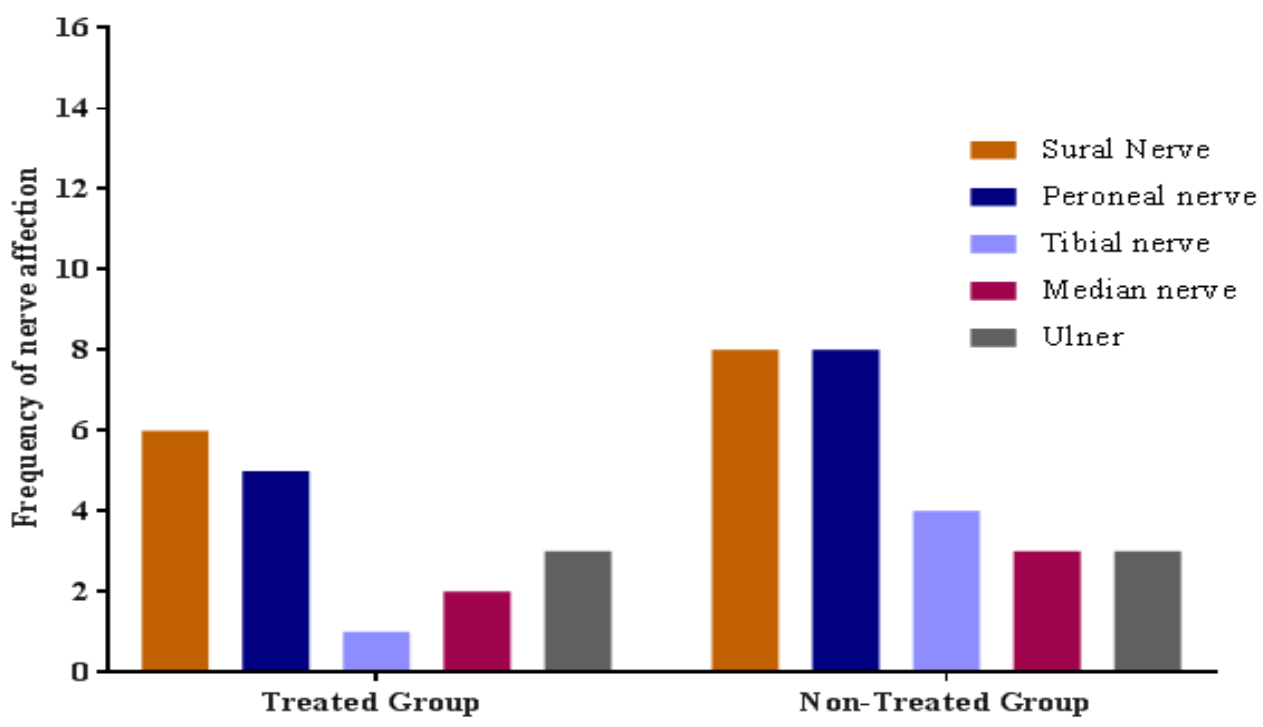

Figure (1): Bar chart displayed the pattern of peripheral neuropathy among patients received colchicine and patients not received colchicine.

Table (3): The pattern of peripheral neuropathy according to colchicine treatment

\begin{tabular}{|l|l|l|l|c|}
\hline Variables & $\begin{array}{l}\text { Treated } \\
(\mathrm{N}=9)\end{array}$ & $\begin{array}{l}\text { Non-Treated } \\
(\mathrm{N}=21)\end{array}$ & $\begin{array}{l}\text { Fisher exact } \\
\text { test }\end{array}$ & P-value \\
\hline Peripheral neuropathy & $6(66.6 \%)$ & $10(47.6 \%)$ & 0.0894 & $>0.05$ \\
\hline Sural nerve affection & $6(66.6 \%)$ & $8(38.1 \%)$ & 0.0512 & $>0.05$ \\
\hline Peroneal nerve affection & $5(55.5 \%)$ & $8(38.1 \%)$ & 0.0695 & $>0.05$ \\
\hline Tibial nerve affection & $1(11.1 \%)$ & $4(19 \%)$ & 0.0618 & $>0.05$ \\
\hline Median nerve affection & $2(22.2 \%)$ & $3(14.7 \%)$ & 0.0639 & $>0.05$ \\
\hline Ulnar nerve affection & $3(22.2 \%)$ & $3(14.3 \%)$ & 0.0103 & $>0.05$ \\
\hline
\end{tabular}


Based on the clinical evaluation, only $7(23.33 \%)$ patients revealed evidence of the peripheral neuropathy. However, on nerve conduction studies, extra nine patients were appeared to have subclinical peripheral neuropathy. Hereafter, sural nerve affection was evident in $8(88.8 \%)$ patients among the asymptomatic group, while there were $6(85.7 \%)$ patients among the symptomatic group. Subsequently, three $(42.8 \%)$ and six $(66.6 \%)$ patients had peroneal nerve affection in the symptomatic and the asymptomatic groups, respectively (Figure $\mathbf{2}$ and Table 4).

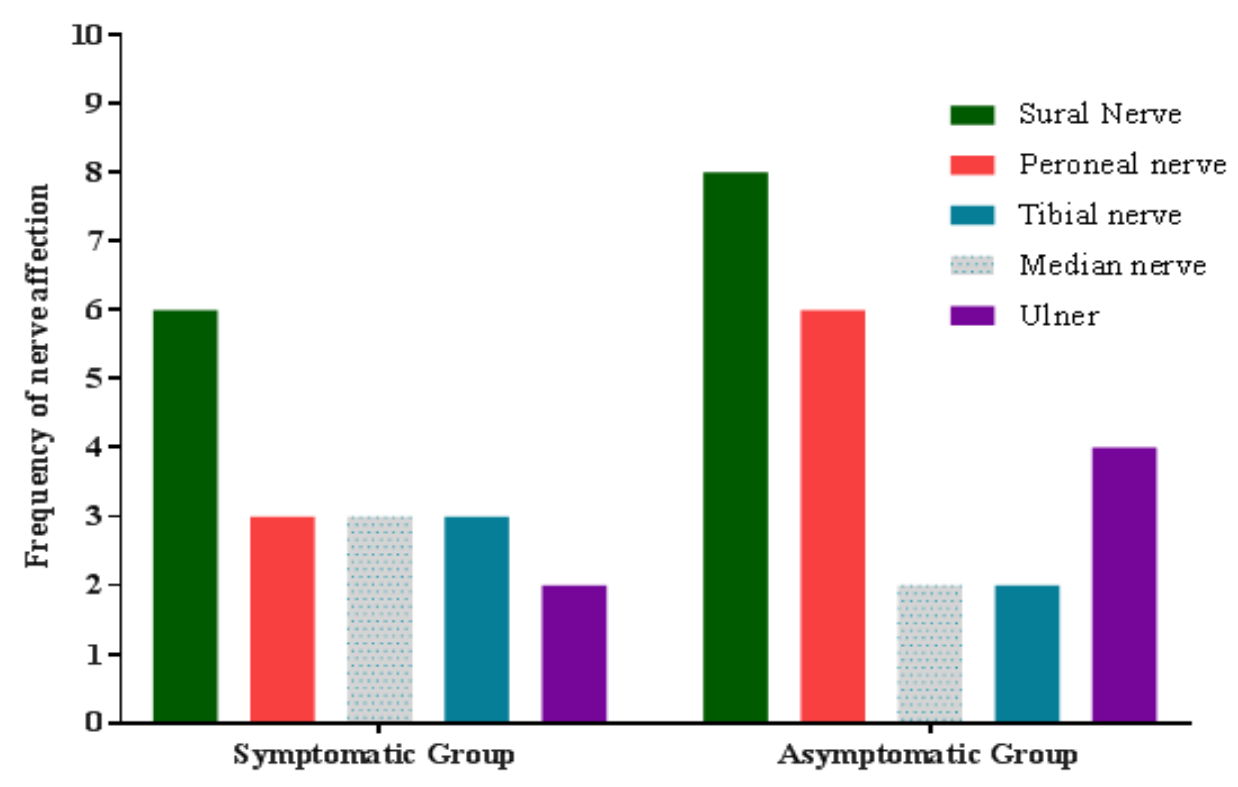

Figure (2): Bar chart showed the pattern of peripheral neuropathy among the symptomatic and asymptomatic groups.

Table (4): The pattern of peripheral neuropathy among the symptomatic and the asymptomatic groups.

\begin{tabular}{|l|l|l|l|l|}
\hline Variables & $\begin{array}{l}\text { Symptomatic } \\
(\mathrm{N}=7)\end{array}$ & $\begin{array}{l}\text { Asymptomatic } \\
(\mathrm{N}=9)\end{array}$ & $\begin{array}{l}\text { Fisher exact } \\
\text { test }\end{array}$ & P-Value \\
\hline Sural nerve & $6(85.7 \%)$ & $8(88.8 \%)$ & 0.0693 & $>0.05$ \\
\hline Peroneal nerve & $3(42.8 \%)$ & $6(66.6 \%)$ & 0.0318 & $>0.05$ \\
\hline Tibial nerve & $3(42.8 \%)$ & $2(22.2 \%)$ & 0.0276 & $>0.05$ \\
\hline Median nerve & $3(42.8 \%)$ & $2(22.2 \%)$ & 0.0489 & $>0.05$ \\
\hline Ulnar nerve & $2(28.5 \%)$ & $4(44.4 \%)$ & 0.0831 & $>0.05$ \\
\hline
\end{tabular}

\section{Correlation and regression analysis}

There was a statistically significant positive correlation between the age of the patients and the incidence of peripheral neuropathy $(\mathrm{r}=0.605$, $\mathrm{p}=0.026)$. Additionally, BDCAF score $(r=0.786, p=0.035)$ and the duration of the disease $(r=0.565, p=0.001)$ showed a statistically significant positive correlation with the occurrence of the peripheral neuropathy. On the other hand, the colchicine treatment had no statistically significant positive correlation with the occurrence of peripheral neuropathy (Figure 3, and Table 5). The univariate regression model revealed that the age of the patients $(\mathrm{p}=0.039)$ and the duration of the disease $(p=0.021)$ are independent predictors of peripheral neuropathy (Figure 4, and Table 6).

There was a statistically significant positive correlation between sural nerve affection and the duration of the 
PERIPHERAL NEUROPATHY IN BEHCET'S DISEASE: CLINICAL...

disease $(\mathrm{r}=0.71, \mathrm{p}=0.005)$ and the BCDAF score $(\mathrm{r}=0.59, \mathrm{p}=0.006)$. Conversely, the peroneal nerve affection was neither correlated with

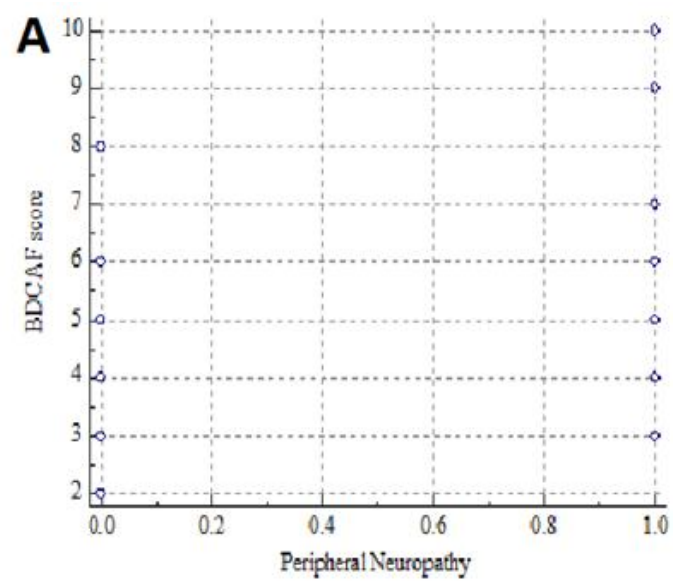

the duration of the disease $(r=-0.01$, $\mathrm{p}=0.93$ ) nor correlated with BCDAF score.

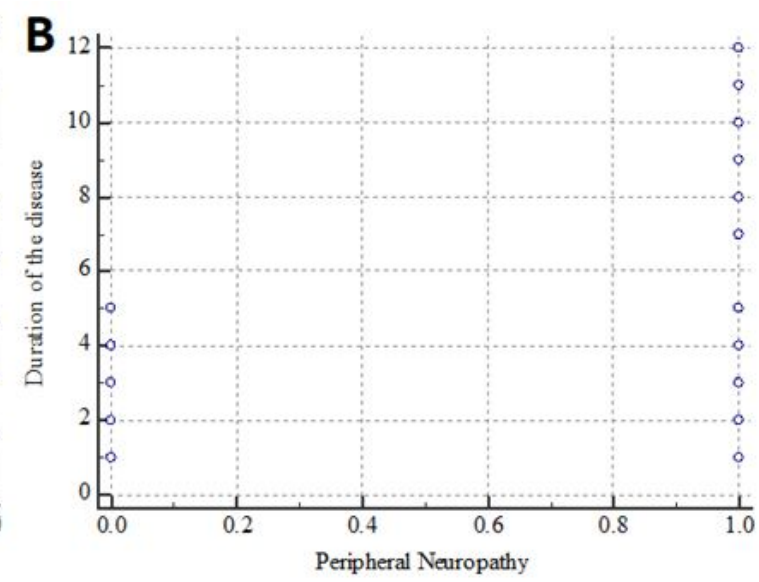

Figure (3): Scatter plot describing the correlation between the occurrence of peripheral neuropathy and A. BDCAF score. B. Duration of the disease
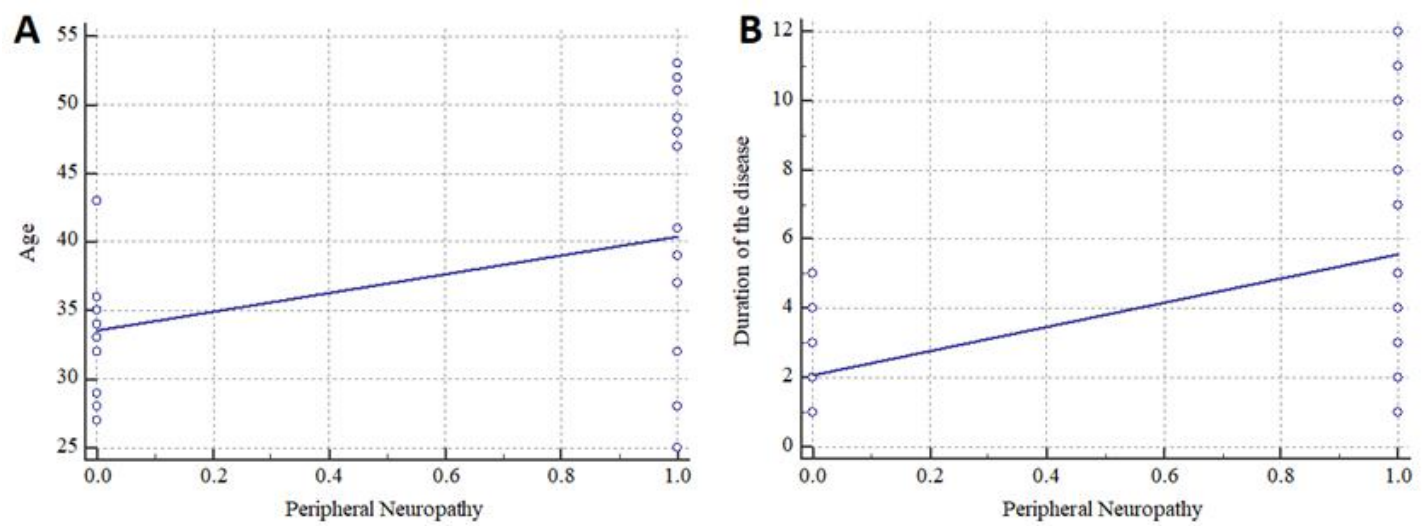

Figure (4): Scatter diagram with regression line illustrated the ability of A. Age and B. duration of the disease in the prediction of peripheral neuropathy

Table (5): Correlations between patients' demographic characteristics and PN

\begin{tabular}{|c|c|c|}
\hline Variable & $\mathrm{r}$ & P-Value* \\
\hline Age & 0.605 & 0.026 \\
\hline Sex & -.146 & 0.44 \\
\hline Duration & 0.565 & 0.001 \\
\hline BDCAF Score & 0.786 & 0.035 \\
\hline Colchicine treatment & -.106 & 0.578 \\
\hline r= Correlation Coefficient, BDCAF= Behcet Disease Current Activity \\
Form, * P-Value based on Spearman's rank correlation coefficient \\
\hline
\end{tabular}


Table (6): Predictors of peripheral neuropathy

\begin{tabular}{|c|c|c|}
\hline \multirow{2}{*}{ Variable } & \multirow{2}{*}{$\beta$} & T-test \\
\cline { 3 - 3 } & & P-Value \\
\hline Age & 0.121 & 0.039 \\
\hline Duration & 0.665 & 0.021 \\
\hline BDCAF Score & 0.522 & 0.053 \\
\hline BDCAF= Behcet Disease Current Activity Form \\
\hline
\end{tabular}

\section{DISCUSSION}

The number of reported patients with neurological insults has been increased. On the contrary, the literature is still doubtful about the true existence of PN along with the possible predictors of such condition among BD patients (Benabdeljlil, 2018 and Caruso \& Moretti, 2018). Thereafter, the present study was carried out to retrieve patients at higher risk to develop PN coupled with the optimal electrophysiological studies, which enhance the early detection of even subclinical PN among BD patients.

The evidence revealed in our investigation brings to light that; even though nearly $20 \%$ of BD patients experienced clinical manifestations of PN, approximately $50 \%$ had PN coupled with demyelinating changes which established electrophysiologically. Thereafter, we supposed to carry out a routine full neurological assessment along with electrophysiological study throughout the follow up period to retrieve BD patients with subclinical PN. Subsequent to that, close follow up protocol should be established, principally for elderly patients, those with long duration of the disease, and patients with high BDCAF score.

The underlying pathogenesis of PN among BD patients includes both humoral and cellular immune-mediated components (Kalra et al., 2014). Noteworthy, the humoral component is evident based on the presence of immunoglobulins gathered with the deposition of the complement on the myelinated nerve fibers (Al-Araji and Kidd, 2009). Subsequently, the cellular immunity role is supported based on the activation of T-cells succeeded by passing of blood-nerve barrier, and the expression of numerous cytokines (Dutra and Barsottini, 2016). These immunological components play a crucial role in $\mathrm{PN}$ development, whereas the manifestations of PN improved considerably among BD patients after receiving immune treatment (Kalra et al., 2014).

Perhaps the significant immunemediated mechanisms, the degradation of nerve axons as an eventual impact of blood vessels damage may be established in order that the activated T-cells are attracted to the nervous system which leads to subsequent nerve ischemia (Shahien and Bowirrat, 2010).

In our study, based on the electrophysiological studies, the pattern of sensory nerve affection was noticeably higher than motor nerve affection. These findings were compatible with Hammad et al. (2014) study who stated that, the sensory and motor nerve conduction studies of ulnar, median, and sural nerves showed a statistically significant 
difference, whereas the sensory affection was considerably more than the motor affection. Similar to that, Akubulat et al. (2007) reported that sensory nerve affection was predominantly higher than motor affection.

Of note, the most affected nerve in current study was sural nerve, whereby approximately half of the included patients showed electrophysiological evidence of affection. This is because the PN among BD patients is a distal polyneuropathy, whereby the lower extremities are predominantly affected, specially the nerves of the lower limbs (Birol et al., 2004). In accordance with our results, Hammad et al. (2014) notified that sural nerve affection was the most frequently involved nerve (50\%) among BD patients.

In our study, the higher age of the patients along with higher BDCAF Score and long duration of symptoms were more susceptible to experience PN. In this respect, Hammad et al. (2014) showed a statically positive correlation between sural and peroneal nerve neuropathy and the duration of the disease.

Although the evidence showed in the current investigation, this study had some limitations apart from the small sample size, lack of randomization, and the short follow-up period which constringed the capability to detect the long term PN consequences among $\mathrm{BD}$ patients. To overcome these limitations, further randomized clinical trials with a considerable sample size along with longterm follow up protocol should be implemented to tackle the obstacles of the current study.

\section{CONCLUSION}

Peripheral neuropathy is a considerable complication of $\mathrm{BD}$, whereby health care providers should employ a routine follow up protocol for early detection of the subclinical PN. Additionally, more concern should be given for elderly patients with high BDCAF score and a long duration of symptoms.

\section{REFERENCES}

1. Akbulut, L., Gur, G., Bodur, H., Alli, N. and Borman, P. (2007): Peripheral neuropathy in Behçet disease: an electroneurophysiological study. Clinical Rheumatology, 26(8): 1240-1244.

2. Al-Araji, A. and Kidd, D. P. (2009): NeuroBehçet's disease: epidemiology, clinical characteristics, and management. The Lancet Neurology, 8(2): 192-204.

3. Benabdeljlil, M. (2018): Neurological complications of Behcet's disease. European Journal of Neurology, 25: 664-664.

4. Birol, A., Ulkatan, S., Koçak, M. and Erkek, E. (2004): Peripheral Neuropathy in Behçet's Disease. The Journal of Dermatology, 31(6): 455-459.

5. Caruso, P., and Moretti, R. (2018): Focus on neuro-Behcet's disease: A review. Neurology India, 66(6): 1619.

6. Dutra, L. and Barsottini, O. (2016): NeuroBehçet's Disease: A Review of Neurological Manifestations and Its Treatment. J Vasc, 2(112): 2 .

7. Dutra, L. A., Goncalves, C. R., BragaNeto, P., Pedroso, J. L., Gabbai, A. A., Barsottini, O. G. and de Souza, A. W. (2012): Atypical manifestations in Brazilian patients with neuro-Behcet's disease. J Neurol, 259(6): 1159-1165.

8. Emmi, G., Bettiol, A., Silvestri, E., Di Scala, G., Becatti, M., Fiorillo, C. and Prisco, D. (2018): Vascular Behçet's syndrome: an update. Internal and Emergency Medicine, 14(5):645-652. 
9. Graham, D., McCarthy, A., Kavanagh, E., O'Rourke, K. and Lynch, T. (2013): Teaching NeuroImages: longitudinally extensive transverse myelitis in neuro-Behcet disease. Neurology, 80(18): e189-190.

10. Hammad, M. A. H., Sharaf, D. M., ElShafey, A. M. and Nasr, M. M. (2014): Peripheral nerve involvement in Behcet's disease; an electrophysiological study. The Egyptian Rheumatologist, 36(4): 195-199.

11. Kalra, S., Silman, A., Akman-Demir, G., Bohlega, S., Borhani-Haghighi, A., Constantinescu, C. S. and Sfikakis, P. P. (2014): Diagnosis and management of Neuro-Behçet's disease: international consensus recommendations. Journal of Neurology, 261(9): 1662-1676.

12. Mazzoccoli, G., Matarangolo, A., Rubino, R., Inglese, M. and De Cata, A. (2016): Behçet syndrome: from pathogenesis to novel therapies. Clinical and Experimental Medicine, 16(1): 1-12.

13. Saip, S., Akman-Demir, G. and Siva, A. (2014): Neuro-Behçet syndrome. In: Handbook of clinical neurology. Pbl. Elsevier, USA, Vol. 121: 1703-1723.

14. Shahien, R. and Bowirrat, A. (2010): Neuro-Behçet's disease: A report of sixteen patients. Neuropsychiatric disease and treatment, 6, 219-225.

15. Siva, A. and Saip, S. (2009): The spectrum of nervous system involvement in Behcet's syndrome and its differential diagnosis. J Neurol. 256(4): 513-529.

16. Tankisi, H., Pugdahl, K., FuglsangFrederiksen, A., Johnsen, B., De Carvalho, M., Fawcett, P. R. and Schofield, I. S. (2005): Pathophysiology inferred from electrodiagnostic nerve tests and classification of polyneuropathies. Suggested guidelines. Clinical Neurophysiology, 116(7): 1571-1580.

17. Uygunoglu, U., Saip, S., and Siva, A. (2018): Behçet's Disease and Neuro-Behçet's Syndrome. Neurology, 6(1):77-85.

18. Yesilot, N., Shehu, M., Oktem-Tanor, O., Serdaroglu, P. and Akman-Demir, G. (2006): Silent neurological involvement in Behcet's disease. Clin Exp Rheumatol., 24(5 Suppl 42): S65-70.

19. Yusuf Yazici, M., Sebahattin Yurdakul, M., and Hasan Yazici, M. (2009): Behçet's Syndrome An Update. International Journal Of Advance, 19 :712-521. 


\section{الاعتلال العصبى الطرفى في مرض بهست: در اسة سريرية ووظيفية عصبية فرفية}

حسام محمد إمام - محي الدين ثروت محمد - حاتم جلال عبد الله* والسيا ماهر عرفات أقسام طب المخ والأعصاب والروماتيزم * ، كلية الطب ـ جامعة الأزهر ـ القاهرة

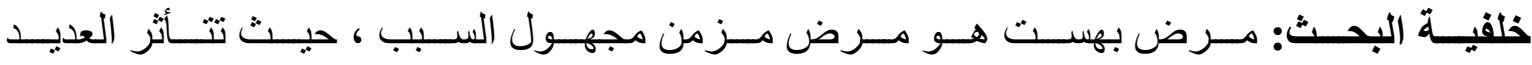

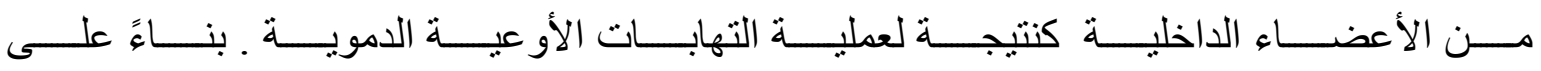

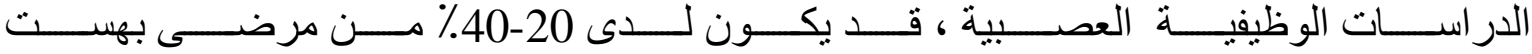

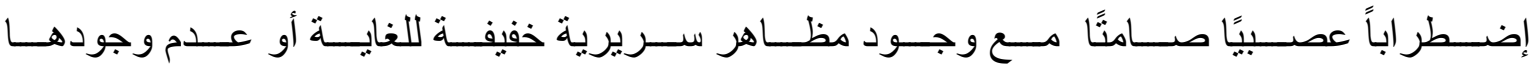
تماماً.

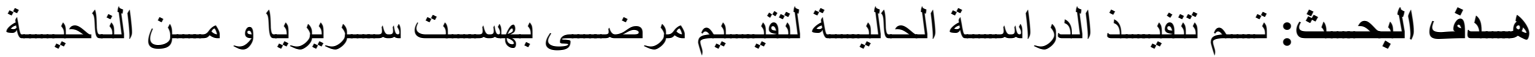

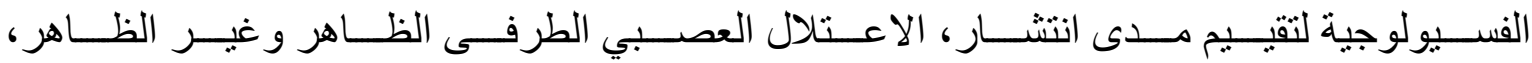
مع تحديد الأسباب التى قد تؤدى إلى وجودة.

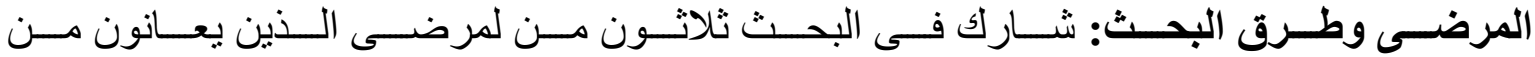

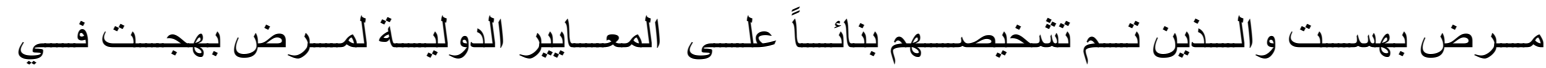

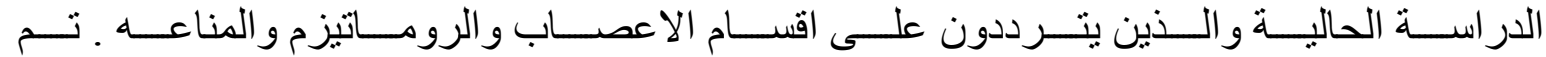

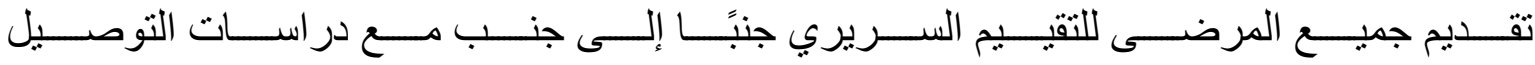

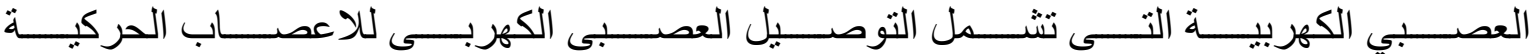

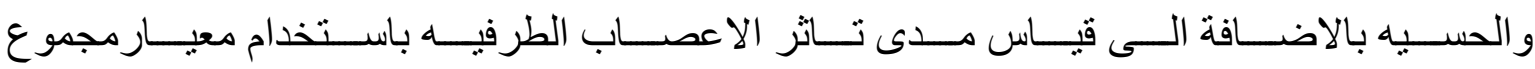
نقاط الاعتلال العصبي.

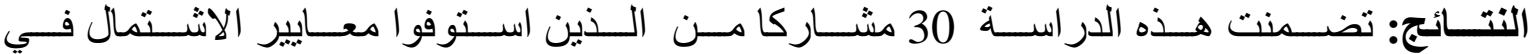

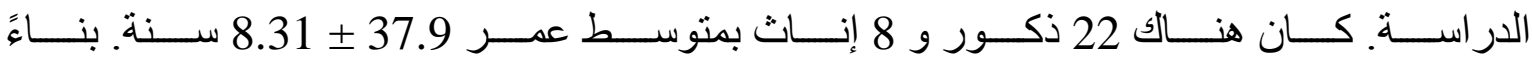

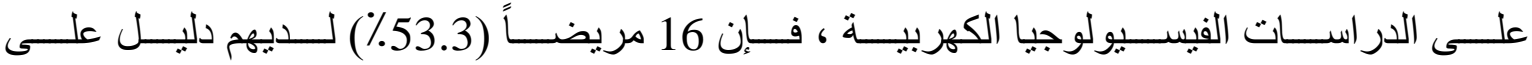

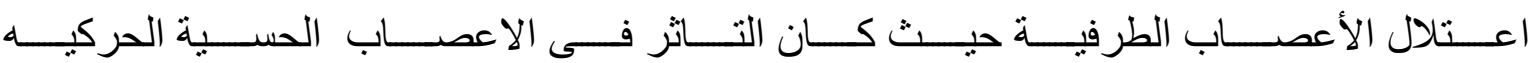

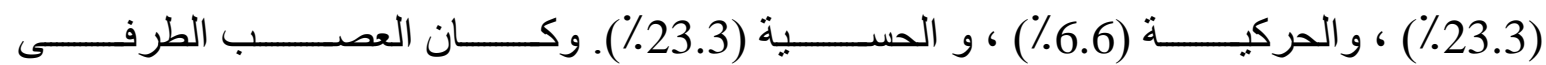




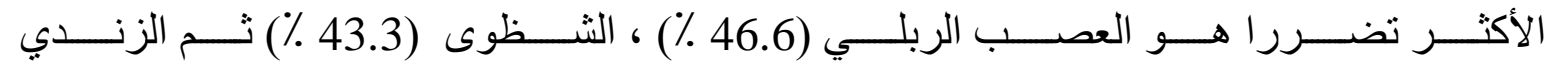

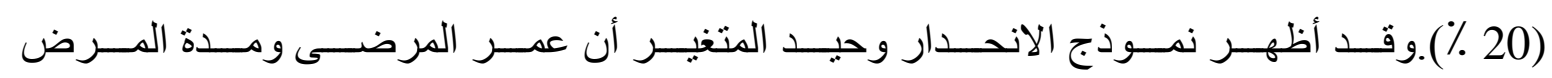
منبئين مستقلين للاعتلال العصبي الطرفى.

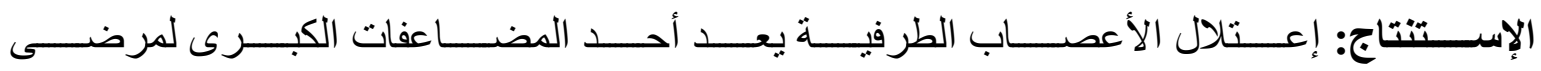

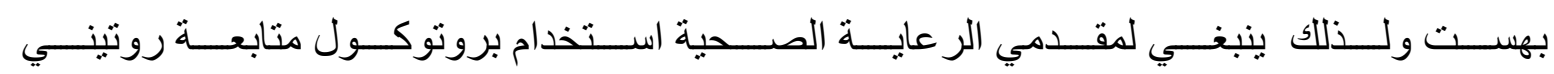
للكثف المبكر عن هذا الإعتلال لتجنب المشكلة المحتملة له. 